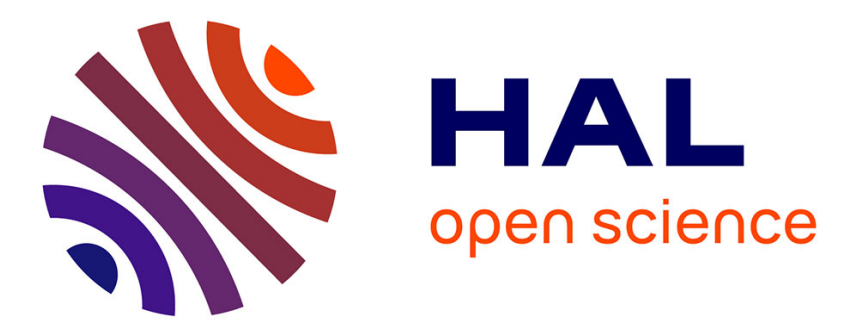

\title{
Quantification de la charge d'entraînement imposée au footballeur professionnel en phase de réhabilitation athlétique post-blessure
}

A. Ben Belgith, Said Ahmaidi, P. Maille, Philippe Noirez, François-Denis

Desgorces

\section{To cite this version:}

A. Ben Belgith, Said Ahmaidi, P. Maille, Philippe Noirez, François-Denis Desgorces. Quantification de la charge d'entraînement imposée au footballeur professionnel en phase de réhabilitation athlétique post-blessure. Science \& Sports, 2012, 27 (3), pp.169-174. 10.1016/j.scispo.2011.06.012 . hal01826191

\section{HAL Id: hal-01826191 \\ https://hal-insep.archives-ouvertes.fr/hal-01826191}

Submitted on 29 Jun 2018

HAL is a multi-disciplinary open access archive for the deposit and dissemination of scientific research documents, whether they are published or not. The documents may come from teaching and research institutions in France or abroad, or from public or private research centers.
L'archive ouverte pluridisciplinaire HAL, est destinée au dépôt et à la diffusion de documents scientifiques de niveau recherche, publiés ou non, émanant des établissements d'enseignement et de recherche français ou étrangers, des laboratoires publics ou privés. 


\title{
Quantification de la charge d'entraînement imposée au footballeur professionnel en phase de réhabilitation athlétique post-blessure
}

\author{
A. Ben Belgith ${ }^{a}$, S. Ahmaidi ${ }^{a}$, P. Maille ${ }^{b}$, P. Noirez ${ }^{c, d}$, F.-D. Desgorces ${ }^{c, e, *}$ \\ a EA-3300, adaptations physiologiques à l'exercice et réadaptation à l'effort, UFR-STAPS, université de Picardie \\ Jules-Vernes, avenue Paul-Claudel, 80025 Amiens cedex, France \\ ${ }^{\mathrm{b}}$ Centre technique national Fernand-Sastre, 78120 Clairefontaine-en-Yvelines, France \\ c UFR-STAPS, université Paris Descartes, 1, rue Lacretelle, 75015 Paris, France \\ ${ }^{\text {d }}$ EA-4466, stress cellulaire : physiopathologie, stratégies nutritionnelles et thérapeutiques innovantes, faculté de \\ pharmacie, université Paris Descartes, 75005 Paris, France \\ e Institut de recherche biomédicale et d’épidémiologie du sport, INSEP, 75012 Paris, France \\ * Auteur correspondant : francois.desgorces@parisdescartes.fr
}

Article publié dans : Science \& sports (ISSN 0765-1597), 2012, vol. 27, n³, pp. 169-174 (doi : 10.1016/j.scispo.2011.06.012)

\begin{abstract}
Résumé
L'objectif de notre travail a été de comparer les méthodes de quantification de la charge d'entraînement «Travail-Endurance-Récupération », « séance-CR10 » et Training impulse lors d'une période de réhabilitation athlétique chez le footballeur professionnel.
\end{abstract}

Sujets et méthodes : onze jeunes footballeurs (16,0 $\pm 1,2$ ans) blessés parmi les 90 jeunes footballeurs fréquentant le Centre technique national Clairefontaine de la Fédération française de football ont fait l'objet d'un recueil de données au cours de leur réhabilitation athlétique post-blessure. Cette période de réhabilitation comprend des séances de renforcement musculaire, de travail des appuis puis de travail individuel avec ballon.

Résultats: Pour l'ensemble des séances, les charges Travail-Endurance-Récupération ne sont pas reliées aux charges séance-CR10 ( $p=0,11)$. Pour les séances d'appui et avec ballon, des relations significatives apparaissent entre les charges Travail-Endurance-Récupération et séance-CR10 $\left(\mathrm{R}^{2}=0,60\right)$, entre Travail-Endurance-Récupération et Training impulse $\left(\mathrm{R}^{2}=0,62\right)$ et entre séanceCR10 et Training impulse $\left(\mathrm{R}^{2}=0,66\right)$. L'évolution des charges des séances de renforcement diffère entre les méthodes Travail-Endurance-Récupération et séance-CR10. Ces différences résident dans le fait que l'augmentation de l'intensité de travail à volume constant se traduit par une baisse de la perception de l'effort. Les conditions très particulières du renforcement musculaire post-blessure font apparaître des limites quant à l'utilisation de la méthode séance-CR10 lors de la première phase de réhabilitation. La méthode Travail-Endurance-Récupération semble utilisable pour l'ensemble des phases de travail.

Conclusion : Notre étude souligne la faisabilité de la quantification de la charge d'entraînement des périodes de réhabilitation athlétique post-blessure ce qui devrait permettre d'optimiser les programmes proposés.

Mots clés: Réadaptation ; Charge d'entraînement; Perception de l'effort; Travail-EnduranceRécupération 


\section{Summary}

The purpose of this study is to compare training load quantification methods "Work-EnduranceRecovery", "session-CR10" and "Training impulse" during a period of post-injury physical rehabilitation in professional soccer players.

Methods: Eleven young soccer players injured participated in the study. This period of physical rehabilitation is composed of sessions of muscle conditioning, proprioception and individual work with ball. For all training sessions, Work-Endurance-Recovery training loads are not related to session-CR10 $(P=0.11)$. These two types of training loads result in significant correlations for proprioception and ball sessions $\left(\mathrm{R}^{2}=0.60\right)$. In addition, significant correlation coefficients appear between TRIMPs and WER training loads $\left(\mathrm{R}^{2}=0.62\right.$ ), and between TRIMPs (only calculated for proprioception and ball sessions) and session-CR10 $\left(\mathrm{R}^{2}=0.66\right)$. Quantification of muscle conditioning sessions differs between Work-Endurance-Recovery and session-CR10 methods. These differences are due to the fact that the increase in exercise intensity associated with unchanged volume results in decreased effort perception. The particular context of muscular conditioning after injury underlines the limits of session-CR10 quantification method used during the first phase of a physical rehabilitation period. The WER method seems useable for all phases of physical rehabilitation. Our study underlines the feasibility of training load quantification for physical rehabilitation exercises after injuries that could allow to improve such exercises programs.

Keywords : Readaptation; Training loads; Perceived exertion; Work-Endurance-Recovery

\section{Introduction}

Le football professionnel moderne impose à l'organisme des charges importantes aux niveaux énergétique, musculaire, tendineux et ligamentaire. Cette sollicitation peut expliquer en partie l’importante fréquence des blessures rapportées par la littérature [1]. Cependant, le risque de blessure peut être limité grâce à des programmes de prévention et à des prises en charges individualisées lors des périodes de réhabilitation post-blessures [1]. La phase de réhabilitation athlétique post-blessure apparaît, par conséquent, comme une étape importante pour l'optimisation de la récupération du sportif et pour préserver son intégrité physique future. Tout comme pour l'entraînement habituel, la notion de charge d'entraînement (CE) pourrait être un outil central permettant de gérer individuellement le travail réalisé lors des périodes de réhabilitation. La CE représente le stress physiologique induit par un exercice sur un athlète [2]. La méthode des Training impulses (TRIMP) proposée au cours des années 1970 a servi de référence et de point de départ à de nombreux travaux réalisés par la suite [3]. Les CE exprimées en TRIMP sont basées sur la durée et l'augmentation de la fréquence cardiaque (FC) de la séance, ce qui suggère une utilisation exclusive de cette méthode pour quantifier les exercices d'endurance [3] and [4]. Par comparaison avec les CE issues de la méthode TRIMP, il a été proposé une méthode de quantification de la CE utilisant la perception de l'effort et la durée de séance [5]. Les CE issues de cette méthode appelée séance-CR10 étaient corrélées avec les CE calculées en TRIMP et notamment lors de séances d'entraînement en football [5] and [6]. L'utilisation de l'échelle de perception de l'effort (CR10) par la méthode séance-CR10 semble prometteuse dans la mesure où la perception de l'effort est rapportée comme étant reliée à l'intensité et/ou au volume d'exercice. Cependant, lorsque des séances de sprint, d'endurance ou de musculation réalisées jusqu’à épuisement ont été comparées, les CE de la séance d'endurance calculées avec la méthode séanceCR10 étaient deux fois plus importantes que celles des séances de sprint et de musculation [4]. Lors de cette étude, une méthode de quantification appelée Travail-EnduranceRécupération basée sur le ratio travail cumulé/travail limite (WER) était proposée. Cette 
méthode visait à fournir des CE similaires pour les exercices intermittents qu'ils soient réalisés à intensité sous- ou supra-maximale [4].

L'objectif de notre travail était d'analyser les charges d'entraînement issues des méthodes WER, séance-CR10 et TRIMP pour des périodes de réhabilitation chez de jeunes footballeurs.

\section{Méthode}

\subsection{Sujets}

Onze jeunes footballeurs (16,0 \pm 1,2 ans) blessés parmi les 90 jeunes footballeurs fréquentant le Centre technique national Clairefontaine de la Fédération française de football ont fait l'objet d'un recueil de données au cours de leur réhabilitation athlétique post-blessure. Ce groupe était constitué de quatre adolescentes et sept adolescents présentant des moyennes de stature de $167 \pm 6 \mathrm{~cm}$, de masse corporelle de 58,3 \pm 8,2 kg et de vitesse maximale aérobie, en début de saison, de $14,3 \pm 1,5 \mathrm{~km} / \mathrm{h}$. N’ont été sélectionnées que les blessures des membres inférieurs (élongations ou déchirures ischio-jambiers ou quadriceps et/ou articulaires dont syndrome rotulien et instabilité du genou) induisant un arrêt de la pratique supérieur à trois semaines avant la réhabilitation athlétique.

\subsection{Protocole du programme de réhabilitation}

La première phase de réhabilitation était composée de cinq séances de renforcement musculaire sur appareil iso-cinétique précédées par un échauffement de dix minutes sur bicyclette ergométrique. La deuxième phase était composée de trois séances de travail des appuis sans ballon progressivement orientées vers des appuis spécifiques au football (les exercices, d'abord réalisés en condition statique, deviennent de plus en plus dynamiques). La troisième phase évaluée était composée de deux séances de travail individuel avec ballon débutant par des exercices simples et allant vers des situations réelles de football (enchaînements déplacement-tirs par exemple).

\subsection{Paramètres mesurés et matériel utilisé}

Lors de chaque séance, le travail cumulé (TC) à l'intensité cible d'exercice et la fréquence d'exercice (ratio travail/récupération) ont été enregistrés. Pour les séances de renforcement, le volume de TC était représenté par le nombre de répétitions réalisé à la puissance développée lors des séances. La puissance développée était enregistrée à partir de l'appareil iso-cinétique (Cybex International, Cybex 6000, Medway, États-Unis). Pour les séances de travail des appuis et de travail avec ballon, le volume de travail était représenté par l'ensemble du temps de travail effectif et l'intensité exprimée par la fraction de la FC de réserve sollicitée lors des périodes effectives de travail.

L'endurance limite individuelle à l'intensité cible d'exercice a été estimé à l'aide de l'échelle ETL (estimation du temps limite) proposée par Garcin et al. [7] qui semble permettre une estimation correcte du temps limite à partir d'exercices réalisés à intensité constante chez des sportifs de bon niveau [8]. Dans ce contexte de réhabilitation post-blessure, l'estimation de l'endurance limite par l'échelle ETL nous semblait préférable à l'utilisation d'équations de prédiction ou à une évaluation des capacités physiques maximales. 
L'échelle de perception de l'effort « Category ratio-10 » (CR-10) utilisée pour notre travail, était connue des participants puisqu'elle est utilisée lors des tests d'effort de début de saison. L'utilisation de l'échelle a été expliquée aux sujets lors de leur inclusion pour l'étude, la semaine précédant le début du programme de réhabilitation. Trente minutes après la fin de chaque séance, les sujets cotaient la séance qu'ils venaient de réaliser de 0 " pas d'effort du tout » à 10 « effort maximal » [9].

La FC des séances de travail d’appui et avec ballon a été mesurée en continu puis moyennée grâce à des cardio-fréquencemètres (Polar Vantage ; Kempele, Finlande) et comparée à la FC maximale $\left(\mathrm{FC}_{\max }\right)$ et de repos $\left(\mathrm{FC}_{\text {repos }}\right)$ mesurées lors d'un test d'effort maximal réalisé sur tapis roulant en début de saison.

\subsection{Quantification de la charge d'entraînement}

Pour quantifier la CE, la méthode WER utilise le TC à l'intensité d'exercice comparé à l'endurance limite (End lim) à cette même intensité lors d'un travail continu, ces éléments étant fonction de la fréquence d'exercice (ratio TC/temps de récupération : TC/TR) de la séance [4]. Le calcul des CE a été réalisé grâce à l'équation suivante :

$$
\mathrm{CE}=\mathrm{TC} / \text { End } \lim +\ln (1+\mathrm{TC} / \mathrm{TR})
$$

Les CE ont été également calculées un utilisant la méthode séance-CR10 qui prend en compte la perception de l'effort et la durée totale de la séance (en minutes), en appliquant l'équation suivante [5] :

$$
\mathrm{CE}=\text { durée } \times \mathrm{CR}-10
$$

Enfin, pour les séances d'appuis et de travail avec ballon, la durée de la séance (DS en minutes) et la $\mathrm{FC}$ de réserve moyenne de la séance $\left[\mathrm{FC}_{\text {réserve }}=\left(\mathrm{FC}_{\text {moyenne }}\right.\right.$ $\left.\left.\mathrm{FC}_{\text {repos }}\right) /\left(\mathrm{FC}_{\text {maximale }}-\mathrm{FC}_{\text {repos }}\right)\right]$ ont été utilisées pour calculer les TRIMP selon les équations suivantes [3] :

$$
\begin{aligned}
& \mathrm{CE}_{\text {TRIMP }}=\mathrm{DS} \times \mathrm{FC}_{\text {réserve }} \times 0,64 \mathrm{e}^{\left(1,92 \times \mathrm{FC}_{\text {réserve }}\right)} \text {, pour les adolescents } \\
& \mathrm{CE}_{\text {TRIMP }}=\mathrm{DS} \times \mathrm{FC}_{\text {réserve }} \times 0,86 \mathrm{e}^{\left(1,67 \times \mathrm{FC}_{\text {réserve }}\right)} \text {, pour les adolescentes. }
\end{aligned}
$$

\subsection{Analyse statistique}

Les indicateurs d'intensités, de volume, de perception de l'effort et de CE ont été comparés grâce à une analyse de variance à deux facteurs (séance $\times$ type d'exercice physique) et mesures répétées (sujet $\times$ séance). Lorsqu'un effet significatif était observé, un test post-hoc de Schéffé était utilisé. Une analyse de régression linéaire a permis d'évaluer les relations entre les différents paramètres recueillis. Les analyses statistiques ont été réalisées en utilisant le logiciel Statistica (version 7.1, Statsoft, Maisons-Alfort, France). Le seuil de significativité statistique a été fixé à $p<0,05$.

\section{Résultats}

Le Tableau 1 présente les volumes, intensités et cotations de la perception de l'effort de chacune des séances réalisées. Une augmentation significative des intensités de travail est 
observée entre chaque séance et celle qui précède, ou qui suit, $(p<0,05)$ hormis entre les séances 3 et $4(p=0,41)$ et les séances 4 et 5 de renforcement musculaire $(p=0,71)$. Cette augmentation régulière de l'intensité s'observe alors que le volume de travail est stable (renforcement musculaire) ou significativement augmenté (travail des appuis et avec ballon ; $p<0,05$ ). De plus, la perception de l'effort est relativement stable mais significativement plus basse en séance 5 qu'en séance 1 de renforcement musculaire $(p=0,04)$. La perception de l'effort est également différente entre la première et la troisième séance de travail des appuis $(p<0,05)$.

Ainsi, lors du renforcement musculaire, les CE WER sont plus hautes en séances 4 et 5 qu'en séance $1(p<0,01)$ et les CE séance-CR10 sont plus basses en séance 5 qu'en séance 1 $(p=0,04)$ (Fig. 1). Lors de la phase de travail des appuis, pour chaque méthode de quantification, la $\mathrm{CE}$ de la séance 3 est significativement supérieure à celle de la séance 1 $(p<0,05)$. Lors de la phase de travail avec ballon, pour les méthodes séance-CR10 et TRIMP, la CE de la séance 2 est supérieure à celle de la séance $1(p<0,05)$.

Pour l'ensemble des séances, les CE WER ne sont pas reliées aux CE séance-CR10 ( $p=0,11)$, ces deux types de charges étant reliés pour les phases de travail des appuis et de travail avec ballon $\left(\mathrm{R}^{2}=0,60, p=0,01\right.$; Fig. 2). De plus, pour ces dernières deux phases, les CE WER $\left(\mathrm{R}^{2}=0,62\right.$; $p=0,01)$ sont reliées aux CE TRIMP et les CE séance-CR10 sont reliées aux CE TRIMP $\left(\mathrm{R}^{2}=0,66\right.$; $p=0,01)$. Les valeurs de CR-10 ne sont pas reliées à l'intensité relative $(p=0,07)$, à la durée $(p=0,08)$ et aux charges d'entraînement calculées $(p>0,05)$. Des relations significatives entre CR10 et intensité relative, durée totale de travail et charges WER sont observées seulement pour les séances d'appui et de travail avec ballon (respectivement, $\mathrm{R}^{2}=0,33 ; \mathrm{R}^{2}=0,31 ; \mathrm{R}^{2}=0,57 ; p<0,05$ ).

\section{Discussion}

Nos résultats montrent une différence de quantification des séances réalisées selon la méthode utilisée et une absence de relation entre les méthodes WER et séance-CR10 lorsque l'ensemble des séances est considéré. Cette absence de relation semble induite par les CE de la phase de renforcement musculaire qui augmentent sur certaines séances avec la méthode WER alors qu'elles diminuent avec la méthode séance-CR10. Entre la première et la cinquième séance de renforcement, la perception de l'effort (composant de la méthode séance-CR10) est abaissée alors que la puissance relative augmente graduellement, que le volume relatif augmente et que la durée de la séance reste stable. Ce résultat est inverse à l'ensemble des études observant la relation entre perception de l'effort et niveau de force relative et/ou volume de travail chez des sportifs ou des sédentaires [10] and [11]. Cependant, nos résultats sont en accord avec des baisses de perception de l'effort, pour un travail physique inchangé, observées chez des patients après arthroplastie de la hanche (après six semaines) ou lors de répétitions d'exercices après infarctus [12] and [13]. Ces travaux et nos résultats confirment une forte influence du niveau de forme, ou des conditions de pratique, sur la perception de l'effort [14] and [15]. Cette baisse progressive de la perception de l'effort lors de la reprise du travail musculaire pourrait s'expliquer par :

- une amélioration rapide des capacités physiques des sujets faisant suite au déconditionnement lié à la blessure ;

- une surestimation de l'effort lors des toutes premières séance, cette surestimation pouvant être liée à une crainte de se blesser à nouveau ou à l'inactivité physique associé à la blessure [16]. 
À l'inverse, nous observons des relations entre les trois méthodes de quantification de la CE pour les phases de travail des appuis et de travail avec ballon. Les méthodes séance-CR10 et TRIMP utilisent un composant commun (durée totale d'exercice) dont les augmentations de séance à séance sont significatives. Ces augmentations de la durée d'exercice s'observent alors que le pourcentage de FC de réserve (composant de la méthode TRIMP) est en augmentation significative lors de certaines séances et que les rares variations de perception de l'effort (composant de la méthode séance-CR10) observées sont des baisses. Par conséquent, la relation observée entre les CE issues des méthodes séance-CR10 et TRIMP semble fortement dépendante de la durée totale d'exercice. Les relations observées entre les charges issues de ces deux dernières méthodes avec celles provenant de la méthode WER suggèrent des interactions entre le niveau d'épuisement atteint (WER), la perception de l'effort (séance-CR10) et le niveau de sollicitation cardio-respiratoire (TRIMP). Ces résultats sont en accord avec des travaux récents associant le volume relatif de travail, la FC et la perception de l'effort [15] and [17].

Nos résultats suggèrent que la prise en compte de la perception de l'effort lors du renforcement musculaire post-blessure mérite une attention particulière, à l'inverse, une utilisation rationnelle de cette perception comme indicateur de CE semble nécessiter davantage d'investigations. De manière similaire au travail de Desgorces et al. [4], la méthode WER semble utilisable pour l'ensemble des phases de travail. Ainsi, notre étude souligne la faisabilité de la quantification de la CE lors de la programmation des exercices de réhabilitation athlétique post-blessure. Cette quantification fiable devrait permettre une meilleure gestion, individualisée et efficace, de ces programmes d'exercices.

\section{Déclaration d'intérêts}

Les auteurs déclarent ne pas avoir de conflits d’intérêts en relation avec cet article.

\section{Références}

[1] M. Hägglund, M. Waldén, J. Ekstrand. Injury incidence and distribution in elite football - a prospective study of the Danish and the Swedish top divisions. Scand J Med Sci Sports, 15 (2005), pp. $21-28$

[2] T. Taha, S.G. Thomas. Systems modelling of the relationship between training and performance. Sports Med, 33 (2003), pp. 1061-1073

[3] E.W. Banister, T.W. Calvert, M.V. Savage, T.M. Bach. A system model of training for athletic performance. Australian J Sports Med, 7 (1975), pp. 57-61

[4] F.D. Desgorces, X. Senegas, J. Garcia, L. Decker, P. Noirez. Methods to quantify intermittent exercises. Appl Physiol Nutr Metab, 32 (2007), pp. 762-769

[5] C. Foster, J.A. Florhaug, J. Franklin, L. Gottschall, L.A. Hrovatin, S. Parker et al. A new approach to monitoring exercise training. J Strength Cond Res, 15 (2001), pp. 109-115

[6] F.M. Impellizzeri, E. Rampinini, A.J. Coutts, A. Sassi, S.M. Marcora . Use of RPE-based training load in soccer. Med Sci Sport Exerc, 36 (2004), pp. 1042-1047

[7] M. Garcin, J.F. Vautier, H. Vandewalle, H. Monod. Ratings of perceived exertion (RPE) as an index of aerobic endurance during local and general exercise. Ergonomics, 41 (1998), pp. 1105-1115 
[8] M. Garcin, J. Coquart, S. Robin, R. Matran. Prediction of time to exhaustion in competitive cyclists from a perceptually based scale. J Strength Cond Res, 25 (2011), pp. 1393-1399

[9] B.J. Noble, G.A. Borg, I. Jacobs, R. Ceci, P. Kaiser A category-ratio perceived exertion scale: relationship to blood and muscle lactates and heart rate. Med Sci Sports Exerc, 15 (1983), pp. 523-528

[10] M.L. Day, M.R. McGuigan, G. Brice, C. Foster. Monitoring exercise intensity during resistance training using the session RPE scale. J Strength Cond Res, 18 (2004), pp. 353-358

[11] R.F. Gearhart, F.L. Goss, K.M. Lagally, J.M. Jakicic, J. Gallagher, K.I. Gallagher et al. Ratings of perceived exertion in active muscle during high-intensity and low-intensity resistance exercise. J Strength Cond Res, 16 (2002), pp. 87-91

[12] C.C. Grange, J. Maire, A. Groslambert, N. Tordi, B. Dugue, J.N. Pernin et al. Perceived exertion and rehabilitation with arm crank in elderly patients after total hip arthroplasty: a preliminary study. J Rehab Res Develop, 41 (2004), pp. 611-620

[13] J.P. Buckley, J. Sim, R.G. Eston. Reproducibility of ratings of perceived exertion soon after myocardial infarction: responses in the stress-testing clinic and the rehabilitation gymnasium. Ergonomics, 52 (2009), pp. 421-427

[14] R. Eston, J. Faulkner, A. St Clair Gibson, T. Noakes, G. Parfitt The effect of antecedent fatiguing activity on the relationship between perceived exertion and physiological activity during a constant load exercise task. Psychophysiol, 44 (2007), pp. 779-786

[15] T. Joseph, B. Jonhson, R.A. Battista, G. Wright, C. Dodge, J.P. Porcari et al. Perception of fatigue during simulated competition. Med Sci Sports Exerc, 40 (2008), pp. 381-386

[16] J.W.S. Vlaeyen, A.M.J. Kole-Snijders, R.G.B. Boeren, H. Van Eek. Fear of movement/(re)injury in chronic low back pain and its relation to behavioural performance. Pain, 62 (1995), pp. 363-372

[17] T.D. Noakes. RPE as a predictor of the duration of exercise that remains until exhaustion. Brit J Sports Med, 42 (2008), pp. 623-624 
Tableau 1. Caractéristiques des séances d'exercice en fonction des phases de réhabilitation athlétique.

\begin{tabular}{|c|c|c|c|c|c|c|c|c|c|}
\hline & \multicolumn{3}{|c|}{ Renforcement musculaire } & \multicolumn{3}{|c|}{ Travail des appuis } & \multicolumn{3}{|c|}{ Travail spécifique avec ballon } \\
\hline & $\begin{array}{l}\text { Volume } \\
\text { (rep) }\end{array}$ & $\begin{array}{l}\text { Intensité } \quad(\%) \\
\text { Pmax) }\end{array}$ & CR-10 & $\begin{array}{l}\text { Volume } \\
\text { (min) }\end{array}$ & \begin{tabular}{|l|} 
Intensité (\% \\
FC $_{\text {res }}$ )
\end{tabular} & CR-10 & $\begin{array}{l}\text { Volume } \\
\text { (min) }\end{array}$ & $\begin{array}{l}\text { Intensité } \\
\left(\% \mathrm{FC}_{\mathrm{res}}\right)\end{array}$ & CR-10 \\
\hline S1 & 49 & $64,7 \pm 3,4$ & $4,9 \pm 1,5$ & $17,5 \pm 2,6$ & $30,5 \pm 7$ & $3,4 \pm 0,5$ & $30,0 \pm 7,1$ & $41,8 \pm 5$ & $3,0 \pm 0,5$ \\
\hline S & 49 & $71,4 \pm 3,8^{\mathrm{a} \text { and } \mathrm{b}}$ & $4,6 \pm 1,8$ & $\begin{array}{l}21,5 \pm 4,2^{a} \\
\text { and } b\end{array}$ & $38,6 \pm 6^{\mathrm{a} \text { and } \mathrm{b}}$ & $3,0 \pm 0,6$ & $\begin{array}{l}38,3 \pm 4,4^{a} \\
\text { and } b\end{array}$ & $44,6 \pm 2$ & $2,8 \pm 0,4$ \\
\hline S4 & 49 & $84,4 \pm 2,9^{b}$ & $4,3 \pm 1,9$ & & & & & & \\
\hline S5 & 49 & $86,7 \pm 2,9^{b}$ & $3,8 \pm 2,1^{b}$ & & & & & & \\
\hline
\end{tabular}

Volume en nombre de répétitions (rep) pour le renforcement musculaire et en minutes (min) pour les deux autres phases; intensité en pourcentage de puissance maximale (\% Pmax) pour le renforcement musculaire et en pourcentage de la fréquence cardiaque de réserve ( $\left.\% \mathrm{FC}_{\mathrm{res}}\right)$ pour les deux autres phases ; perception de l'effort selon l'échelle CR-10 (cotation de 0 à 10).

a Significativement différent de la séance précédente.

b Significativement différent de S1 de la phase de réhabilitation considérée. 
Figure 1. Évolution des charges d'entraînement selon la méthode Travail-Endurance-Récupération (WER), la méthode séance-CR10 et la méthode des training impulses (TRIMP) pour les séances 1 à 5 de renforcement (renf1 à renf5), pour les séances 1 à 3 d'appui (app1 à app 3) et les séances 1 et 2 de travail avec ballon (ball 1 et ball 2). * : significativement différent de renf1; \# : significativement différent d’app1 ; \$ : significativement différent de ball 1.
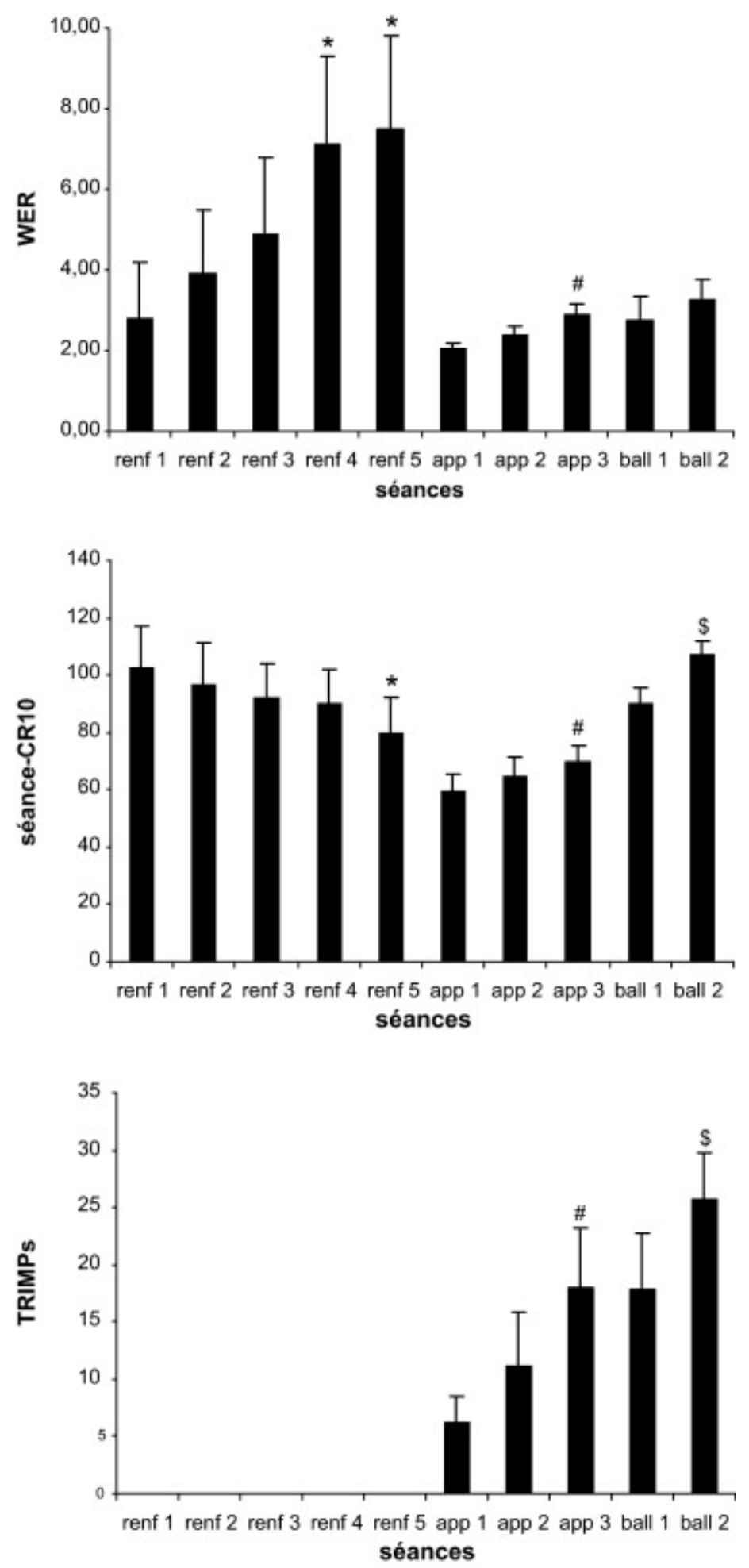
Figure 2. Relations entre les charges d'entraînement calculées pour les séances de travail des appuis et avec ballon à partir des méthodes Travail-Endurance-Récupération (WER), séance-CR10 et training impulses (TRIMP).
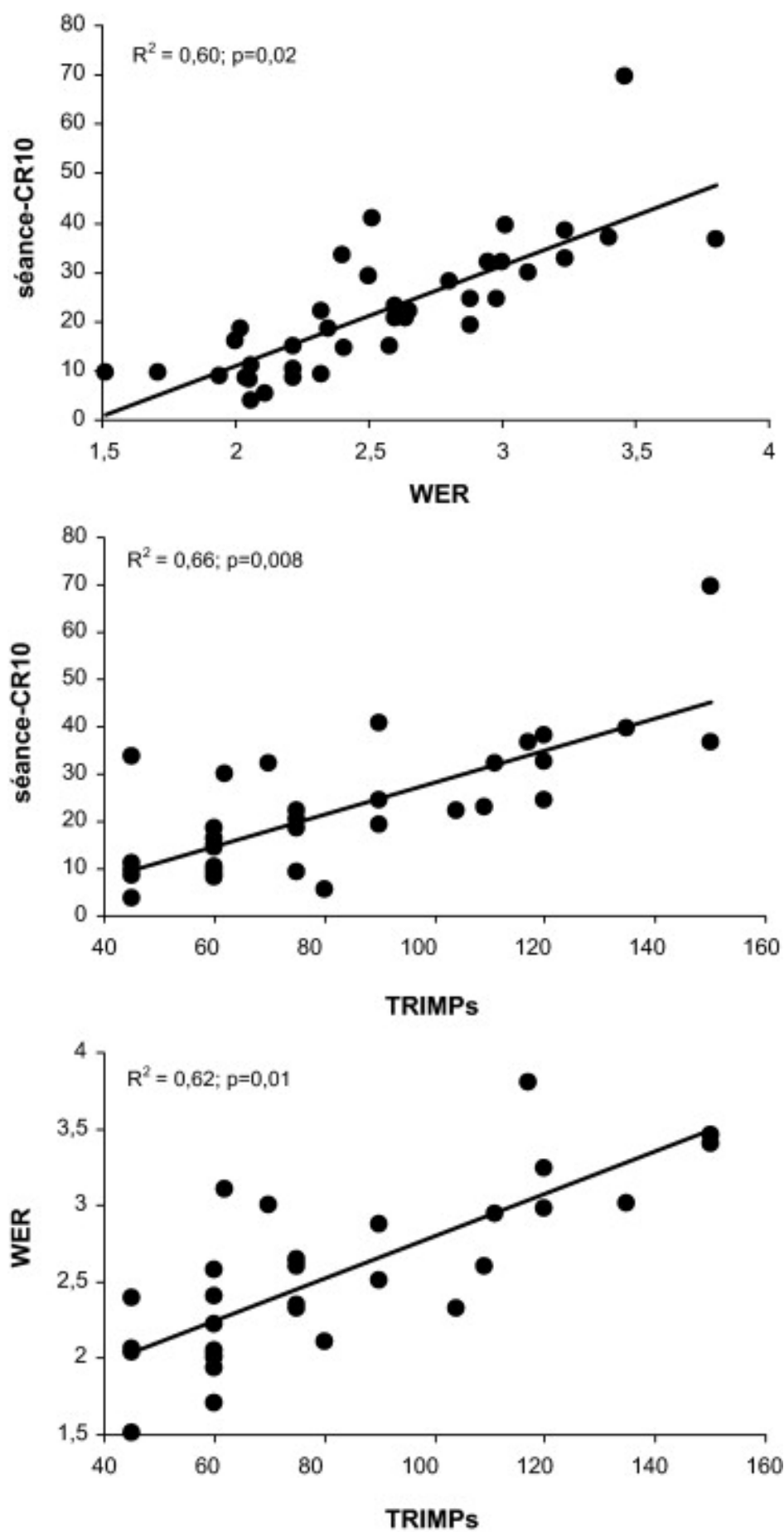